\title{
Injection Integrity in 2020
}

\author{
James Crowley, MHA, CNMT
}

A agents and therapies, the injection process and results should be carefully considered. In today's complex medical delivery system, referring and treating physicians often have little or no understanding of the potential impact of suboptimal dose delivery on examination accuracy. Learning from over 3,000 injections in PET/CT and nuclear medicine at Carilion Clinic in Roanoke, VA, has led us to just as many questions as answers. The assumptions in nuclear medicine are profound, and the purpose of this Practical Protocol Tip is to discuss techniques, potential improvements, and thoughts on what happens when we inject radioactive material.

On May 14, 1980, the Federal Register (volume 45, number 95, page 31703) was updated to document that an extravasation or infiltration is not considered a misadministration: "Extravasation is the infiltration of injected fluid into the tissue surrounding a vein or artery. Extravasation frequently occurs in otherwise normal intravenous or intraarterial injections. It is virtually impossible to avoid. Therefore, the Commission does not consider extravasation to be a misadministration." The effect of this position is that there are no requirements that ordering physicians or patients be informed if there is a minor or significant infiltration of a radiopharmaceutical injection. Much has changed in nuclear medicine and molecular imaging in the last $40 \mathrm{y}$. Precise quantitation relies on the assumption that the dose intended was the dose delivered. An incomplete injection has the potential of impacting not only image quality but image quantitation as well. In this patient-satisfactiondriven era, regardless of whether we believe infiltration matters quantitatively or simply results in suboptimal image quality, our patients deserve for us to learn and do better.

\section{DO INFILTRATIONS MATTER IN ${ }^{18}$ F-FDG PET/CT? YES, NO, AND MAYBE}

No. If the interpreting physician does not report SUV measurements, then infiltrations have little to no impact on the results presented.

Maybe. Regardless of whether the interpreting physician interprets the SUV, if the infiltration is moderate to severe there have been several case series on the impact of SUV and image quality. More study and review are needed.

Yes. Institutions that report SUV measurements should review publications on the impact of infiltrations on SUV measurements. There is strong evidence that if moderate to severe infiltration occurs, SUVs either should not be reported by the interpreting physician without comment or should not be reported at all.

COPYRIGHT (C) 2020 by the Society of Nuclear Medicine and Molecular Imaging.
Interpreting physicians should be aware of injections outside the imaging field of view and the possibility of dose stasis in the venous system after injection. In the test-retest setting or baseline follow-up, one should ensure that interval measures of change are real by replicating examination input parameters such as uptake time, scanning method, and dose delivery method.

\section{DO INFILTRATIONS MATTER IN NUCLEAR MEDICINE (NOT THERAPY)? YES, NO, AND MAYBE}

No. General nuclear medicine would largely seem not to be impacted by infiltrations.

Maybe. Moderate to severe infiltrations have not been studied enough to determine. Often the location being imaged is outside the area of infiltration, leaving too many unknowns to determine.

Yes. When attempting quantification, we have identified issues. Careful consideration is needed to understand the dose and the timing of the injection into the bloodstream.

For myocardial perfusion imaging, it is imperative that for the 1-d protocol in which a low-dose resting examination is followed by a high-dose stress examination, the proper lowdose-to-high-dose ratio of 3-3.5 times greater be delivered. An infiltration during the second portion could cause a restrest study as a result of improper or incomplete delivery of the stress dose. Infiltration can lead to a false-negative study even if there truly is underlying cardiac ischemia. This could easily explain how a patient might have normal myocardial perfusion imaging results but a severely abnormal cardiac catheterization.

For bone scans, accurate quantitation relies on complete delivery of the planned dose.

Our facility sought to use data from the Lara System (Lucerno Dynamics) to understand our injection integrity and help staff improve their process in order to decrease human error. We learned that infiltrations happen and, although impossible to completely eradicate, can be significantly reduced. We should not assume we are always doing well and achieving optimal dose injection. We have found it helpful to monitor our performance, use data from the monitoring process to improve performance, and translate this into process improvement. Three things that are completely within one's control and have helped us improve our injection integrity are shown in Table 1.

TABLE 1

\begin{tabular}{cccc}
\hline Infiltration rate & $\begin{array}{c}\text { Measure } \\
\text { phase }\end{array}$ & $\begin{array}{c}\text { Improve } \\
\text { phase }\end{array}$ & $P$ \\
\hline Overall & $13.3 \%$ & $2.9 \%$ & $<0.001$ \\
$\begin{array}{c}\text { For nonantecubital } \\
\text { fossa injection }\end{array}$ & $28.6 \%$ & $7.0 \%$ & 0.0026 \\
$\begin{array}{c}\text { For antecubital } \\
\text { fossa injection }\end{array}$ & $8.5 \%$ & $1.5 \%$ & 0.0039 \\
\hline
\end{tabular}




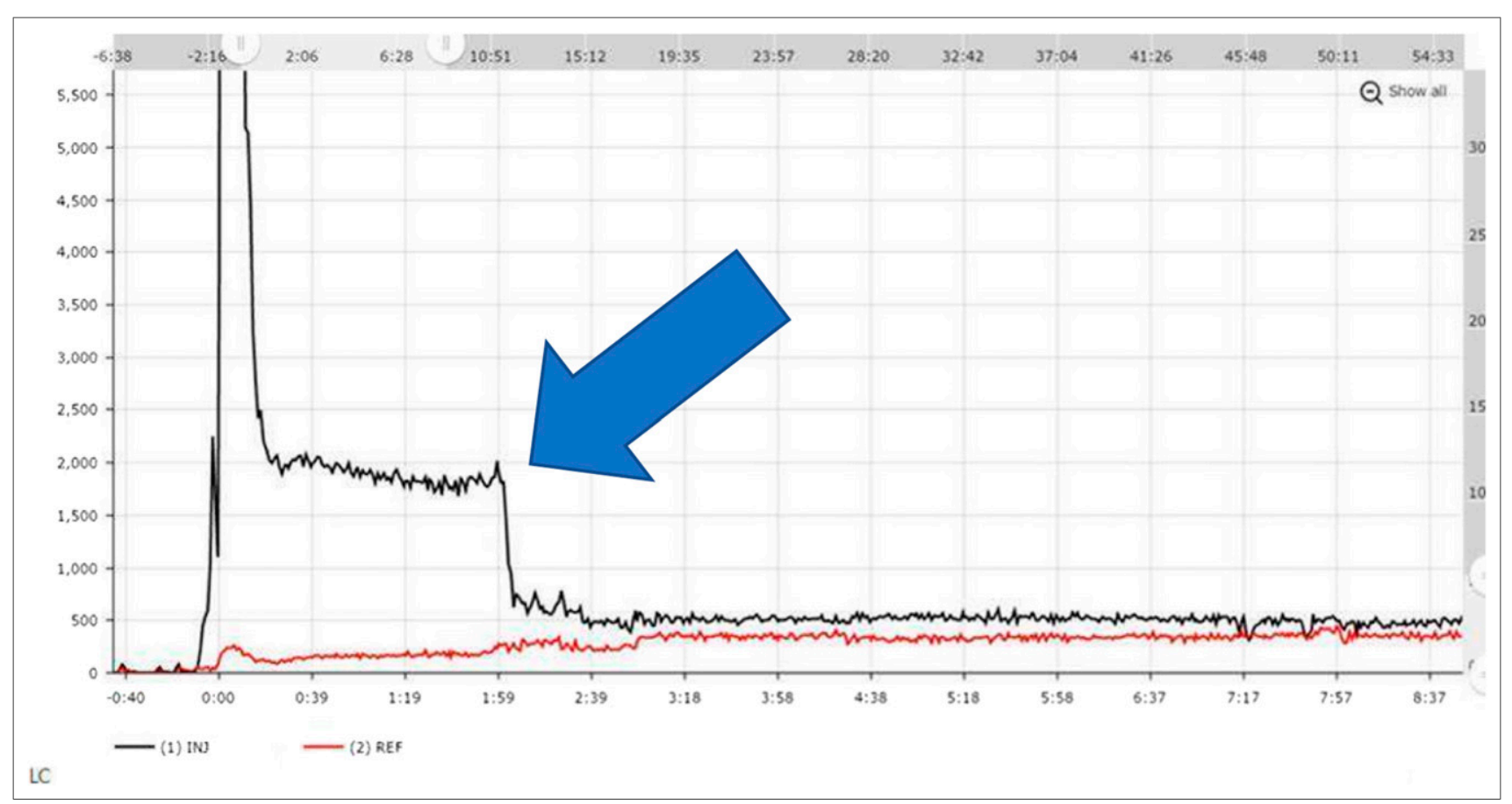

FIGURE 1. Count rates for 2 arms. Arrow shows time point when patient began very light exercise. INJ = injected arm; REF = reference arm.

\section{TECHNICAL CHANGES}

Do Not Use an Intravenous Line That Has Been in Place More Than 24 Hours. In our initial quality assurance and quality improvement project, infiltrations occurred 3 times more often in an indwelling catheter.

Slow the Injection. On the basis of our data from 1,748 consecutive injections, the infiltration rate was $1.7 \%$ when an autoinjector was used and $6.7 \%$ when it was not. Our second study on injection integrity determined that our infusion device had $75 \%$ fewer infiltrations than hand injections. The same techs would establish the intravenous lines and would infuse the saline at a rate up to 10 times faster than the flow rate of the infusion device. After reeducation and learning, we reduced the infiltration rate to $3.5 \%$. We need to remember that we are injecting live tissue that we have punctured. We currently try to infuse at a rate of $1 \mathrm{~mL} / \mathrm{s}$ to mimic our infusion device.

Understand and Teach the Use of Data to Improve Performance in the Workplace. All staff members who have been able to see their data have been able to improve. Understanding areas in which they excel and areas where improvement is needed has allowed technologists to learn and work with peers to do better.

Understand the Impact of Stasis and Whether We Should Continue to Have Patients Hold Still After Injection. Figure 1 demonstrates the count rates for 2 arms and the phenomenon of stasis. Tracer stasis in the arm was seen in nearly $10 \%$ of all patients reviewed for infiltration on PET/CT. Our hypothesis is that movement of the arms and body activates the muscles, increasing blood pressure and allowing the natural lymphatic and vessel flow to help circulate the tracer. The time of stasis varies widely, and stasis was a common phenomenon at sites participating in quality assurance and quality improvement testing on infiltrations. Stasis represents a potential confounding factor in the path to quantification if the tracer is assumed to be available at time zero. Although guidelines recommend no movement after injection, we have found that simple exercises such as moving the

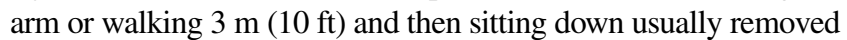
this confounder from our data.

\section{ADDITIONAL TIPS}

Encourage patient hydration before arrival. Avoid straight sticks. For difficult venous access, seek expert assistance (intravenous specialist), consider alternate injection sites (feet or ankles), and use warm packs and the correct needle size.

\section{CONCLUSION}

Infiltrations can be reduced. Using the techniques described, our 2 departments have shown a significant reduction in infiltration rates. Our main department, with a fixed-site PET/CT scanner, began with a $13.3 \%$ infiltration rate and reduced it to under $3 \%$ for 2 consecutive years and continues to improve. The mobile PET/CT department started with a $15.5 \%$ infiltration rate, reduced it to under $5 \%$, and has maintained it there. Nuclear medicine technologists can learn to understand how injections are impacting procedures being performed and advocate to ensure our procedures are of high quality and reproducible.

\section{DISCLOSURE}

James Crowley has received research funds and honoraria for speaking engagements to talk about infiltration and injection integrity from Lucerno Dynamics, the maker of Lara, a system to monitor and support staff learning and education about injections in nuclear medicine. No other potential conflict of interest relevant to this article was reported. 